\title{
Effects of Beta-Hydroxy-Beta-Methylbutyrate Supplementation on Elderly Body Composition and Muscle Strength: A Review of Clinical Trials
}

\author{
Nicolas de Angelus Costa Riela ${ }^{a}$ Maiara Moeme Alvim Guimarães ${ }^{a}$ \\ Daniela Oliveira de Almeida ${ }^{b, c}$ Edilene Maria Queiroz Araujo ${ }^{b, d}$ \\ aLife Sciences Department, University of The State of Bahia (UNEB), Bahia, Brazil; ${ }^{b}$ Life Sciences Department, Nucleus \\ of Research and Extension in Nutritional Genomics and Metabolic Dysfunctions (GENUT), University of The State of \\ Bahia (UNEB), Salvador, Brazil; ' Professor of undergraduate medical course, UniFTC, Salvador, Brazil; ' ${ }^{\text {Life }}$ Sciences \\ Department/UNEB, Health Sciences Institute/UFBA, Coordinator of Nutrition Course and GENUT, Professor at \\ Post-Graduation Program Interactive Process of Organs and Systems, Salvador, Brazil
}

\section{Keywords}

Elderly · Beta-hydroxy-beta-methylbutyrate .

Supplementation · Strength · Muscle mass · Sarcopenia

\begin{abstract}
Background: The aging process has great impact on body composition, such as the increase of adipose tissue in abdominal region, and the decrease of lean body mass, due to skeletal muscle loss. A reduction in muscle mass is associated to high risk of fractures and falls, loss of mobility, and increased number of hospitalizations. Beta-hydroxy-betamethylbutyrate (HMB) is a biological substance derived from leucine metabolism, with anabolic and anticatabolic properties. Some HMB effects are tissue repair stimulation and protein anabolism. Aims: We aimed to evaluate the effects of HMB supplementation on body composition and muscle strength in elderly, as well as to identify the efficient dosages to reach these effects. Methods: This review included studies that evaluated muscle mass and muscle strength, associated or not with physical exercise and diet in elderly people. Only studies published from 2008 to 2019 were selected for analysis. Results: Six articles were included in the review. The used doses varied from 1.5 to $3 \mathrm{~g}$. In 5 studies, HMB supple-
\end{abstract}

mentation was associated with calcium; only 1 study did not use the oral administration route. Two studies used $4 \mathrm{~g}$ of maltodextrin as a vehicle; 1 used HMB with a hypercaloric and hyperproteic supplement; 1 associated HMB with lysine and arginine; and 1 with arginine and glutamine. Supplementation of $3 \mathrm{~g}$ of HMB has shown to be most beneficial in improving strength and body composition in people over 65 years, especially in bed rest and untrained conditions. Conclusion: Our findings suggest that HMB has a positive effect on body composition and strength, especially in bedridden or sedentary elderly, due to its anticatabolic properties.

(c) 2021 S. Karger AG, Basel

\section{Introduction}

During the aging process, several body changes affect quality of life. Aging leads to great impacts on body composition, such as increasing of adipose tissue in the abdominal region, and lean body mass reduction due to the loss of skeletal muscle. These are common biological alterations in senescence. However, when they come to be exacerbated, they may lead to significant functional impairment [1]. 
Fig. 1. Flowchart of articles: search and se-

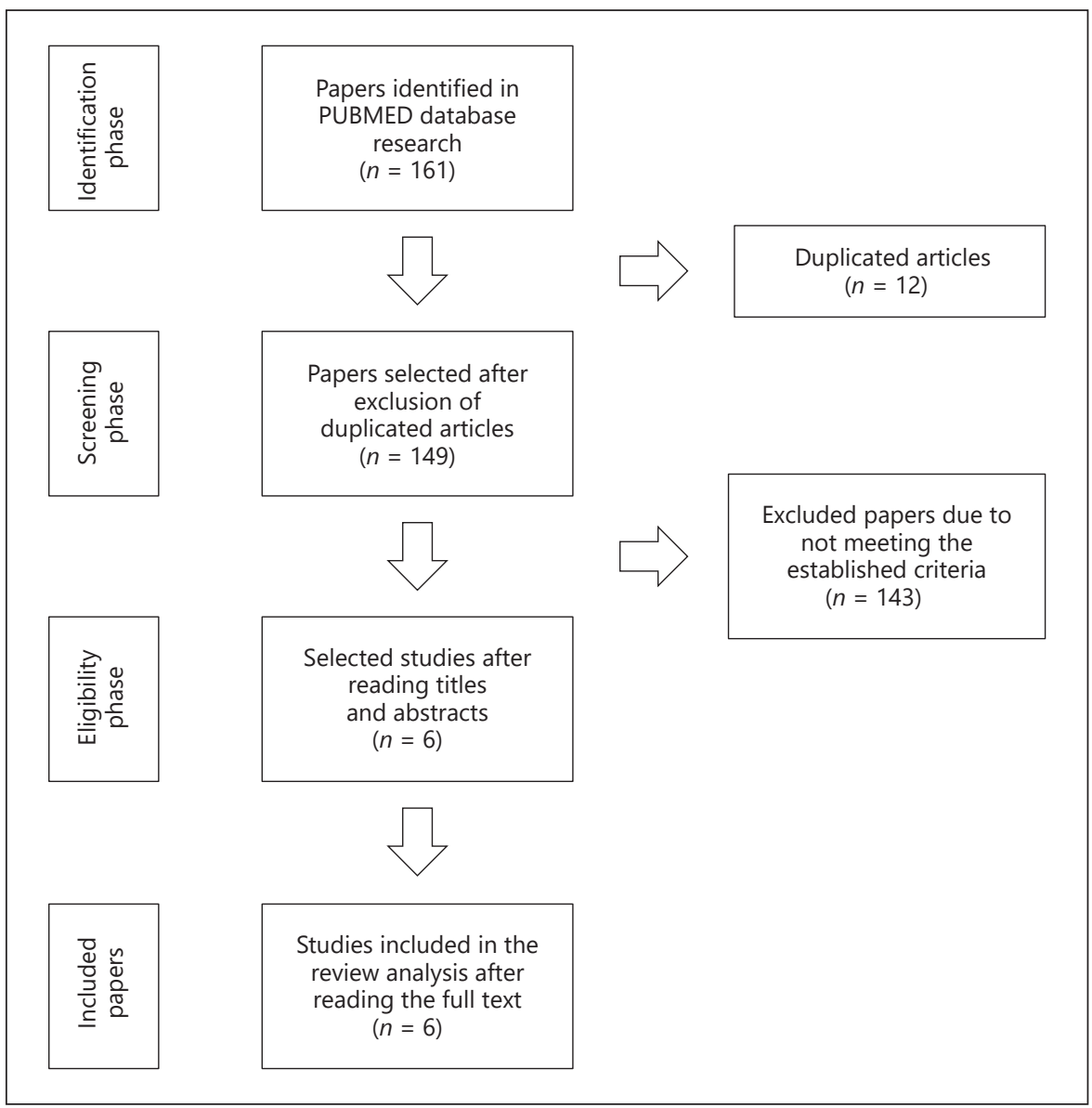

There are inherent physiological changes during senescence due to hormonal dysregulation, associated diseases, mitochondrial dysfunction, hormonal alterations, poor diet, lack of physical activity, and anabolic resistance, which impact straight into the quality of life. In general, all these factors are linked to sarcopenia, as well as alterations in body composition, directly impacting in elderly people autonomy [2-4].

Inadequate levels of muscle mass may lead to higher risk of fractures, falls, loss of mobility, dependence, an increase in the number of hospitalizations, and to a variety of metabolic changes since there are movement limitation and loss of strength $[1,5,6]$. In addition, bedridden elderly people may have muscle atrophy due to disuse, increasing the risk of developing further muscular problems $[7,8]$.

It is widely discussed in scientific literature that regular physical activity and adequate nutritional support are considered safe methods to achieve healthy aging. Some therapeutic and supplemental resources, such as beta-hydroxy-beta-methylbutyrate (HMB), may aid in the prevention of muscle loss reduction during aging. Many supplements have been empirically used for promotion of health in elderly, which brings to light the need for more studies to prove its efficiency $[8,9]$.

HMB is a biological substance derived from leucine metabolism and plays important role in anabolic and anticatabolic processes, such in stimulation of mammalian rampamycin target protein (mTOR) and inhibition of proteolytic processes. Thus, some positive effects on muscle mass and performance, such as catabolic processes reduction, tissue repair stimulation, and anabolism induction, are attributed to HMB [4]. Therefore, the purpose of the review is to verify whether supplementation with $\mathrm{HMB}$ can improve body composition and muscle strength in the elderly people, as well as to identify effective dosages to achieve these effects. 


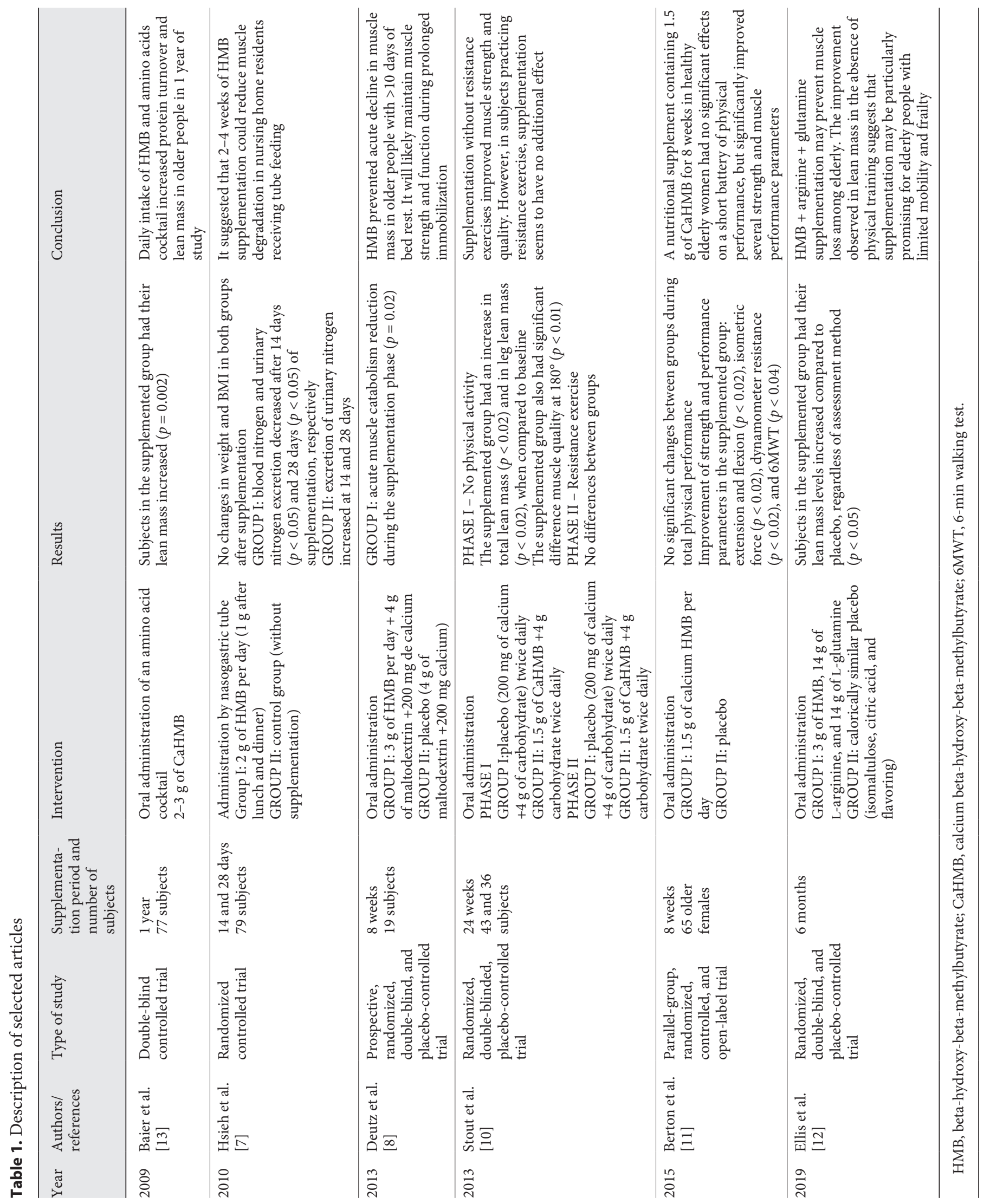




\section{Methods}

It is a review of a collection of clinical trials that analyzed HMB supplementation on strength and body composition in elderly. An initial search in the PUBMED database was performed. Two out of the 9 articles used in the introduction were included in the review $[7,8]$. The words "elderly," "HMB," "beta-hydroxy-betamethylbutyrate," "supplementation," "strength," "muscle mass," and "sarcopenia" were used as keywords for the search of the articles in English language.

Inclusion criteria were publications from 2008 to 2019, including clinical trials studies. Only studies with elderly people that evaluated muscle mass and muscle strength, associated or not with physical exercise and diet, were selected. Animal studies, reviews, case-control cohort studies with subjects under 60 years old, and research that did not assess body composition were not included. Three authors independently searched for articles using the predefined eligibility and exclusion criteria.

\section{Results}

161 articles were found in the initial search in the PUBMED database. After identifying and excluding duplicate articles, 149 articles were selected. In the next step, after reading titles and abstracts, 143 articles were not included due to not fulfilling the pre-established criteria as described in the method section. Finally, 6 articles were selected, and after reading the complete text, they were all being included in this review. Figure 1 illustrates a flowchart of article search and selection, following the preestablished inclusion and exclusion criteria.

Table 1 shows the general effects of HMB supplementation on body composition and physical performance in elderly. It describes the selected articles, compiling information regarding location, type of study, administered dose of $\mathrm{HMB}$, supplementation period, type of intervention, and general effects of HMB supplementation in the studied sample.

Four of the selected articles are North American [8, $10-12$ ], 1 is Italian [13], and 1 is Taiwanese [7]. Four of the studies were conducted with healthy participants [1013], 1 was performed with bedridden elderly people who used nasogastric tube [7], and another was a controlled trial, which confined subjects to voluntary bed rest [8]. All selected studies were conducted with subjects over the age of 65 years, and the intervention periods ranged from 10 days [8] to 1 year [10].

The doses used in the studies varied from $1.5 \mathrm{~g}$ [13] to $3 \mathrm{~g}[8,10,11]$. One study used doses according to each subject's body weight (2 and $3 \mathrm{~g}$ ) [12]. In 5 studies, $\mathrm{HMB}$ supplementation was used as calcium $\operatorname{HMB}[7,8,10,12$,
13]. In 1 study, HMB supplementation was administered via nasogastric tube [7]. In the other 5 studies, HMB supplementation was orally administered. Two studies used $4 \mathrm{~g}$ of maltodextrin as a vehicle $[8,10], 1$ used a hypercaloric and hyperproteic supplement [13], 1 associated $\mathrm{HMB}$ with lysine and arginine [12], and other with arginine and glutamine [11].

The selected studies evaluated body composition and physical performance through different methods. Dualenergy X-ray absorptiometry was the most commonly used approach for body composition assessment, especially lean mass $[8,10,12,13]$. In addition to dual-energy $\mathrm{X}$-ray absorptiometry, 1 study used the bioelectrical impedance analysis to assess cell mass [12], 1 used air displacement plethysmography [11], and 1 added the peripheral quantitative computed tomography to assess muscle density [13]. Only 1 study used body circumferences and BMI as methods for assessing body composition [7]. For evaluation of general physical performance, the preferred method was the short physical performance battery $[8,13]$. For assessment of isolated parameters, such as muscle strength and functionality, the tests used were knee extension and dynamometer [10], the get-upand-go test $[8,10]$, and the 6 -min walking test $(6 \mathrm{MWT})$ [13].

\section{Discussion}

Our study shows that HMB supplementation improves strength and muscle function in elderly. However, there are few clinical trials using HMB supplementation; thus, only 6 articles were used for this analysis. In addition, due to different methodologies, the assessment of $\mathrm{HMB}$ actual effectiveness in attenuating the aging process is rather limited.

Hsieh and colleagues [7] demonstrated that HMB supplementation had a positive effect during an intervention period of 2-4 weeks. Although differences in anthropometric parameters were not significant, the authors found a decrease in blood urea and urinary urea excretion, suggesting protein degradation reduction. Thus, supplementation had an expected anticatabolic effect. However, there are some limitations in this trial, such as the short period of supplementation. Despite its promising results, a longer period may induce more benefits.

Similarly, Deutz and colleagues [8] have shown promising results using $3 \mathrm{~g}$ of $\mathrm{HMB}$ during 10 days of bed rest. Their findings showed that supplementation reduced acute muscle loss during bed rest and preserved strength 
during the rehabilitation period when compared to the control group. This article had a well-designed methodology, since supplementation dose was the conventionally used in the scientific literature, as well as controlled the study subjects and their diets, mitigating methodological biases.

In the abovementioned studies, supplementation had a positive effect on reducing muscle catabolism, which was assessed by biochemical data such as reduced blood urea and urinary urea excretion [7], or by body composition evaluation [8]. Despite different routes of administration, the findings support and validate HMB supplementation, especially in older people that are susceptible to severe muscle loss. According to Holecek [4], elderly have resistance to anabolic signs, and together with muscles disuse, they may lead to severe loss, thus making supplementation a beneficial approach.

When comparing the results found in both articles [7, 8] with the mechanisms of action suggested in literature, it brings to light the positive effects of HMB. Wilson and colleagues [14] synthetized the proposed molecular mechanisms by which HMB may protect skeletal muscle degradation. In their review, they propose that HMB inhibits the expression and activity of the ubiquitin-proteasome system, an irreversible proteolytic system, which is overactivated in situation of movement restriction [14]. Indeed, an in vitro and in vivo study investigated the role of HMB in preventing muscle mass and strength losses in a rat model of muscle atrophy induced by the use of dexamethasone [15]. Even though experimental interventions were performed in animals, their results are promising since oral supplementation of HMB ameliorated body weight and lean mass losses induced by the drug. Authors found that HMB attenuated dexamethasone-induced lysosomal proteolysis and autophagossome formation, as well as regulated ubiquitin activity and the expression of activation factors of the proteasome system [15].

Another point that contributes for HMB supplementation is that it may prevent anabolic resistance in elderly, which is caused by mitochondrial dysfunction, low-grade inflammation, and oxidative stress [4]. Studies show that HMB properties also prompt activation of adenosine monophosphate-activated protein kinase (AMPK kinase) and NAD-dependent sirtuin 1 deacetylase (Sirt 1), which stimulate mitochondrial biogenesis [16], and reduce the expression of tumor necrosis factor receptor 1 (TNFR1), tumor necrosis factor (TNF), angiotensin II, and interleukin-6 receptors [17]. Since TNF and IL-6 pathways are related to systemic low-grade inflammation, which in turn is positively associated to sarcopenia and functional impairment [18], it may explain HMB effects in attenuating muscle loss and frailty in elderly population.

After 24 weeks of experimental period, Stout and colleagues [10] observed a significant increase in strength and in total and leg lean mass in untrained subjects when compared to their respective baseline values. Although the difference between the groups was not statistically significant, the supplemented group had an improvement in muscle quality, a parameter assessed by the relation between strength and the amount of muscle mass. However, it is noteworthy that when individuals performed strength training, there was a change in body composition yet without any significant difference between placebo and supplemented groups. Taken together with the former references $[7,8]$, it is observed that subjects who do not have mechanical stimulation of the musculature or anyone in high susceptibility to muscle loss seem to get more benefits with the use of HMB than trained subjects.

On the other hand, Baier et al. [12] showed that 1 year of HMB supplementation promoted positive effects on lean mass, while the control group had a loss in this parameter. Neither of the groups showed improvement in muscle strength. It was the only article that indicated a supplementation dose based on body weight, and even though HMB supplementation was associated with an amino acid cocktail containing lysine and arginine, the control group also received this cocktail, without the HMB. Ellis et al. [11] also observed changes in body composition in people older than 65 years, when they co-administered $\mathrm{HMB}$, arginine, and glutamine to improvement in lean body mass. They suggest that this positive effect is due to the use of HMB in association with other nutritional sources.

In addition to the aforementioned reduction in muscle catabolism, other possible mechanism of action that explains the results of the studies by Stout et al. [10], Baier et al. [12], and Ellis et al. [11] may be the anabolic properties of $\mathrm{HMB}$, which stimulates the target protein rampamycin in humans (mTOR). The activation of this pathway increases mRNA translation and phosphorylation of 4EBP-1 and p70S6k, which are mTOR effectors. It leads to myofibrillar proteins synthesis, thereby increasing lean mass, which is a desirable effect for the elderly [11].

Our findings are similar to a meta-analysis held in 2015, in which Wu and colleagues [19] concluded that HMB decreases muscle atrophy, especially in people under conditions in which this process is accelerated, such as bedridden patients. In their meta-analysis, there were inconsistent data regarding strength and physical performance parameters. We also found this inconsistence in
Ann Nutr Metab 2021;77:16-22 DOI: $10.1159 / 000514236$
Costa Riela/Alvim Guimarães/Oliveira de Almeida/Araujo 
the studies of our review. In addition, HMB does not seem to have additional effect on body composition when associated with strength training in elderly. One of the possible explanations is due to the inherent stimulation of muscular hypertrophy by this type of training, overcoming the effects of supplementation.

It is noteworthy that there are few studies evaluating supplementation associated with physical activity for a period longer than 6 months, specifically in elderly population. Berton et al. [13] observed that HMB supplementation did not improve performance in a short exercise battery nor body composition in older women. However, the supplemented group had a significant improvement in strength and muscle density. Some limitations of this article were the short experimental period (only 8 weeks) and the low dose of supplementation $(1.5 \mathrm{~g})$. The study population also had high scores on the parameters used to assess performance and strength, and it may explain the lack of positive effects in the supplemented group. In addition, supplementation was co-administered with other substances such as vitamin $\mathrm{D}$, calcium, and protein, and consequently it brings to discussion whether $\mathrm{HMB}$ was the actual agent that caused the improvement of the mentioned parameters. This combination, however, reinforces that we can exploit the synergistic effect of $\mathrm{HMB}$ with other substances.

Considering that muscle tissue is a determining factor for the quality of life in senescence, since it has a direct relationship with balance and strength, $\mathrm{HMB}$ comes to be a potential ally for this public. An advantage of $\mathrm{HMB}$ is that the supplementation had no adverse effects in any of the studies analyzed in our review, regardless of dose or administration route. The absence of negative effects may be an additional factor to encourage its use by the elderly.

\section{Conclusion}

HMB supplementation seems to be a feasible alternative for the maintenance of muscle mass in the population over 65 years, especially in bedridden or sedentary elderly, aiding in the reduction of muscle catabolism. Three grams of HMB seem to be the optimal dose to promote these benefits. Although some studies have shown positive results in short time interval, long-term studies have shown better results in body composition. Thus, a prolonged use of $\mathrm{HMB}$ may promote more important clinical changes.

Few studies accurately assess HMB supplementation in elderly. However, the current findings are promising.

Additional studies are required to provide more information about biological effects of HMB supplementation and hence support HMB use by elderly population.

\section{Acknowledgement}

The authors acknowledge the support of University of State of Bahia for providing devices, infrastructure, and technical support for the implementation of this study.

\section{Statement of Ethics}

Ethics Committee approval was not required since this paper is a systematic review, and no experimental procedure was performed in human beings. Every study included in this systematic review followed the ethical guidelines of the Declaration of Helsinki.

\section{Conflict of Interest Statement}

The authors have no conflicts of interest to declare.

\section{Funding Sources}

This study has no funding source.

\section{Author Contributions}

The contributions of the authors are as follow: N. Riela, M.A. Guimarães, and D. Almeida for searching published papers on the current theme; N. Riela and E. Araujo for designing this study; N. Riela, D. Almeida, and E. Araujo for writing this paper. All authors read and approved the manuscript.

References

Ann Nutr Metab 2021;77:16-22

DOI: $10.1159 / 000514236$
1 Edström E, Altun M, Bergman E, Johnson H, Kullberg S, Ramírez-León V, et al. Factors contributing to neuromuscular impairment and sarcopenia during aging. Physiol Behav. 2007;92(1-2):129-35.

2 Marzetti E, Lees HA, Wohlgemuth SE, Leeuwenburgh C. Sarcopenia of aging: underlying cellular mechanisms and protection by calorie restriction. Biofactors. 2009;35(1):28-35.

3 Woo J. Nutritional interventions in sarcopenia: where do we stand? Curr Opin Clin Nutr Metab Care. 2018;21:19-23.

4 Holeček M. Beta-hydroxy-beta-methylbutyrate supplementation and skeletal muscle in healthy and muscle-wasting conditions. J Cachexia Sarcopenia Muscle. 2017;8(4):529-41. 
5 McPhee JS, French DP, Jackson D, Nazroo J, Pendleton N, Degens H. Physical activity in older age: perspectives for healthy ageing and frailty. Biogerontology. 2016;17(3):567-80.

6 Hunter GR, Singh H, Carter SJ, Bryan DR, Fisher G. Sarcopenia and Its Implications for Metabolic Health. J Obes. 2019;2019:8031705.

7 Hsieh LC, Chow CJ, Chang WC, Liu TH, Chang CK. Effect of beta-hydroxy-betamethylbutyrate on protein metabolism in bed-ridden elderly receiving tube feeding. Asia Pac J Clin Nutr. 2010;19(2):200-8.

8 Deutz NE, Pereira SL, Hays NP, Oliver JS, Edens NK, Evans CM, et al. Effect of $\beta$-hydroxy- $\beta$-methylbutyrate (HMB) on lean body mass during 10 days of bed rest in older adults. Clinical nutrition. 2013;32:704-12.

9 Borack MS, Volpi E. Efficacy and safety of leucine supplementation in the elderly. J Nutr. 2016;146(12):2625S-9S.

10 Stout JR, Smith-Ryan AE, Fukuda DH, Kendall KL, Moon JR, Hoffman JR, et al. Effect of calcium $\quad \beta$-hydroxy- $\beta$-methylbutyrate (CaHMB) with and without resistance training in men and women $65+$ yrs: a randomized, double-blind pilot trial. Exp Gerontol. 2013;48:1303-10.
11 Ellis AC, Hunter GR, Goss AM, Gower BA. Oral supplementation with beta-hydroxy-beta-methylbutyrate, arginine, and glutamine improves lean body mass in healthy older adults. J Diet Suppl. 2019;16(3):281-93.

12 Baier S, Johanssen D, Abumrad N, Rathmacher JA, Nissen S, Flakoll P. Year-long changes in protein metabolism in elderly men and women supplemented with a nutrition cocktail of $\beta$-hydroxy- $\beta$-methylbutyrate (HMB), l-arginine, and l-lysine. JPEN J Parenter Enteral Nutr. 2009;33:71-82.

13 Berton L, Bano G, Carraro S, Veronese N, Pizzato S, Bolzetta F, et al. Effect of oral beta-hydroxy-beta-methylbutyrate (HMB) supplementation on physical performance in healthy old women over 65 years: an open label randomized controlled trial. PLoS One. 2015;10(11):e0141757.

14 Wilson JM, Fitschen PJ, Campbell B, Wilson GJ, Zanchi N, Taylor L, et al. International Society of Sports Nutrition Position Stand: betahydroxy-beta-methylbutyrate (HMB). J Int Soc Sports Nutr. 2013;10(1):6.
15 Girón MD, Vílchez JD, Shreeram S, Salto R, Manzano M, Cabrera E, et al. $\beta$-Hydroxy- $\beta$ methylbutyrate (HMB) normalizes dexamethasone-induced autophagy-lysosomal pathway in skeletal muscle. PLoS One. 2015; 10(2):e0117520.

16 Durkalec-Michalski K, Jeszka J, Podgórski T. The effect of a 12-week beta-hydroxy-betamethylbutyrate (HMB) supplementation on highly-trained combat sports athletes: a randomised, double-blind, placebo-controlled crossover study. Nutrients. 2017;9(7):753.

17 Towsend JR, Fragala MS, Jajtner AR, Gonzalez AM, Wells AJ, Mangine GT, et al. $\beta$-Hydroxy- $\beta$-methylbutyrate $(\mathrm{HMB})$-free acid attenuates circulating TNF- $\alpha$ and TNFR1 receptor expression post-resistance exercise. J Appl Physiol. 2013;115:1173-82.

18 Beyer I, Mets T, Bautmans I. Chronic lowgrade inflammation and age-related sarcopenia. Curr Opin Clin Nutr Metab Care. 2012; 15(1):12-22.

19 Wu H, Xia Y, Jiang J, Du H, Guo X, Liu X, et al. Effect of beta-hydroxy-beta-methylbutyrate supplementation on muscle loss in older adults: a systematic review and meta-analysis. Arch Gerontol Geriatr. 2015;61(2):168-75. 